\title{
QUISTE DEL CONDUCTO COLÉDOCO. REPORTE DE UN CASO
}

COMMON BILE DUCT CYST. CASE REPORT

CISTO DO CONDUTO COLÉDOCO. REPORTE DUM CASO

\section{VANESSA SERPA CUESTA', RENE MENDOZA MERCHÁN', WILSON SOLÓRZANO LOOR'}

\author{
${ }^{1}$ Hospital Clínica Alcívar, Guayaquil, Ecuador
}

\section{Resumen}

El quiste de colédoco es una patología congénita benigna de las vías biliares que se presenta en la infancia e incrementándose en la actualidad en la edad adulta más en el sexo femenino y con especial énfasis en la raza asiática, y su diagnóstico es en base al estudio colangiográfico y la clasificación más aceptada es la de Todani y Cols. Se presenta un caso de una paciente femenina de 26 años de edad, de la ciudad de Guayaquil, que presenta cuadro clínico caracterizado por dolor abdominal más anorexia y vómito. Su diagnóstico se lo hizo por colangiografia y colangioresonancia. El tratamiento fue quirúrgico y se realizó una hepato yeyunostomía en $Y$ de Roux, con una evolución favorable.

PALABRAS CLAVE: quiste, conducto de colédoco, colangiografía, anomalía congénita.

Abstract

The choledochal cyst is a benign congenital bile duct pathology that occurs in childhood and increasing today in the older adult female literacy, with special emphasis on the Asian race, and its diagnosis is based on the study cholangiographic and most accepted classification is Todani et al. We report a case of a female patient of 26 years old in the city of Guayaquil that has the same clinical picture characterized by abdominal pain and vomiting more anorexia. The diagnosis is made by cholangiography and what colangioresonancia. The treatment was surgical and hepato jejunostomy was performed in Roux And with a favorable evolution.

KEYWORDS: cysto, common bile duct, cholangiography, congenital abnormalities

0 cisto do colédoco é uma patologia congénita benigna das vias biliares, que se apresentam na infância e aumenta, atualmente, mais na vida adulta de mulheres, em especial na raça asiática. 0 seu diagnostico é basado no estudo colangiografico e a classificação mais aceitada é a do Todani Y Cols. Se apresenta um caso de uma paciente feminina de 26 anos de idade, da cidade de Guaiaquil, que apresenta dor abdominal, anorexia e vomito. 0 seu diagnostico foi feito pela colangiografia e colangioresonancia. 0 tratamento foi cirúrgico. Um hépato yeyunostomia em Y de Roux foi realizada com uma evolução favorável.

PALABRAS-CHAVE: cistos, ducto colédoco, colangiografia, anormalidades congênitas. 
INTRODUCCIÓN

El quiste de colédoco es una malformación de origen congénito de las vías biliares, siendo más frecuente en la infancia y de usual hallazgo en la edad adulta, afecta alrededor del $1 \%$ de la población en nuestro medio con predominio en el sexo femenino; más común en personas de raza asiática ${ }^{1,2,5,7}$, se asocia a complicaciones como infecciones, neoplasias y obstrucciones. Actualmente se dice que la génesis de esta patología se debe al reflujo de enzimas pancreático-biliares dentro del conducto biliar común por una desembocadura anómala del mismo dentro del conducto pancreático que conlleva a una inflamación, con la consecuente fibrosis y dilatación del conducto colédoco. ${ }^{1-4}$

Para su diagnóstico lo más adecuado, por su fácil acceso y costo, es el ultrasonido de abdomen, además se cuenta con un examen más preciso como la colangiografía. ${ }^{8,10}$ El tratamiento es quirúrgico depende del tipo, tamaño y compromiso del quiste aunque con mayor predominio se realiza la hepato yeyunostomía en $Y$ de Roux. A continuación se presenta un caso clínico de una paciente femenina de 26 años de edad que debuta con dolor abdominal tipo cólico más anorexia y vómito preciso.

CASO CLÍNICO

Paciente femenina de 26 años de edad, nació y actualmente residente en la ciudad de Guayaquil, estado civil casada, raza mestiza; acude a la consulta por presentar desde hace aproximadamente dos meses, cuadro clínico caracterizado por dolor abdominal tipo cólico localizado en hipocondrio derecho de leve a moderada intensidad que se alivia con medicamentos antiespasmódicos y aumenta con la ingesta de alimentos, acompañado de náuseas y anorexia. No tiene antecedentes médicos ni familiares, no fuma ni bebe alcohol. Su historia gineco-obstétrica se resumen en tres gestas con cesárea, sin ningún aborto.

Al examen físico la paciente está orientada en tiempo, espacio y persona, hemodinámicamente estable y sin alteraciones cardiopulmonares. El abdomen se encuentra blando, depresible doloroso a nivel de hipocondrio derecho con ruidos hidroaéreos disminuidos. El resto de órganos y sistemas sin alteración. Se realiza ecografía abdominal que muestra vesícula biliar distendida con abundante microlitiasis y barro biliar. El hemograma reporta ligera leucocitosis de $11000 / \mathrm{cc}$, con $76 \%$ de neutrófilos y enzimas hepáticas con TGP de 67 UI/L, TGO de 82 UI/L, fosfatasa alcalina de $56 \mathrm{UI} / \mathrm{L}$, bilirrubina total de $1.0 \mathrm{mg} / \mathrm{dl}$, bilirrubina indirecta de $04 . \mathrm{mg} /$ dl. bilirrubina directa de $0.6 \mathrm{mg} / \mathrm{dl}$. Las enzimas pancreáticas tenían valores de amilasa a 75 UI y lipasa a 88 UI que no muestran gran alteración, por lo cual se decide realizar colecistectomía laparoscópica.

Durante la intervención se procede a la conversión a cirugía abierta por migración de microcálculos a la vía biliar principal. Se realiza exploración de vía biliar extrayendo gran cantidad de microcálculos y barro biliar sin lograr canalizar el esfínter de Oddi al llegar al duodeno, por lo cual se coloca sonda de Kehr en conducto colédoco y se realiza la colecistectomía. A las 48 horas del post operatorio se realiza colangiografia transKehr obteniendo la imagen que se muestra en la figura 1, evidenciando dilatación de la vía biliar intra y extra hepática y conducto colédoco con un diámetro mayor de $3.7 \mathrm{~cm}$, compatible con quiste de colédoco. Ésto se corrobora con una colangiorresonancia que confirma el diagnóstico de quiste de colédoco Todani I.(Figura 2). ${ }^{1,10}$

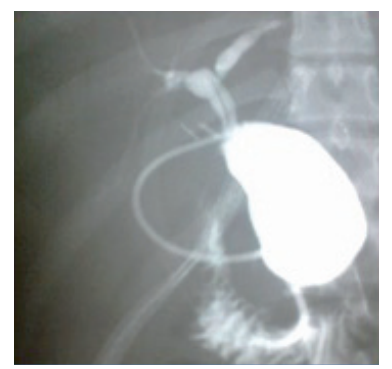

Figura 1. Colangiografía que demuestra dilatación del conducto colédoco con presencia de sonda de Kehr.

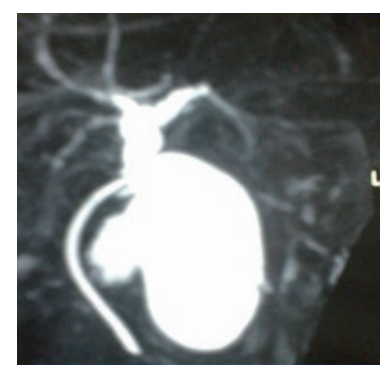

Figura 2. Colangiorresonancia; se evidencia quiste de colédoco de gran tamaño, Todani l.
El informe de resonancia magnética de abdomen reporta imagen compatible con quiste de colédoco tipo I según Todani con diámetro de $3.8 \mathrm{~cm}$ por $3.7 \mathrm{~cm}$, ausencia de vesícula biliar. Bazo, páncreas y riñones de aspecto normal, no se evidencia adenopatías retroperitoneales ni alteración en las paredes abdominales.

Con estos hallazgos se programa una laparotomía exploratoria para exéresis del quiste del colédoco en conjunto a una hepáticoyeyunoanastomosis en Y de Roux (figura 3, 4 y 5). Se realizó anastomosis de asa de yeyuno a $60 \mathrm{~cm}$ del ángulo de Treitz al conducto hepático común con puntos simples perforantes totales de vycril 4-0; la cual no presentó complicaciones. 


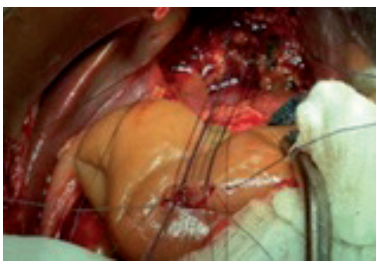

Figura 3. Preparacion del asa del yeyuno a $60 \mathrm{~cm}$ del angulo de treitz y del conducto hepático común para la anastomosis.

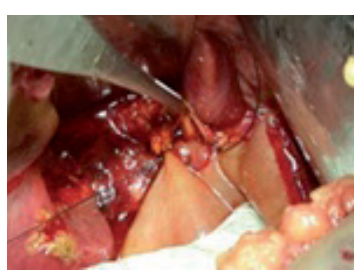

Figura 4. Sutura de la pared posterior de la anastomosis hepático-yeyuno.

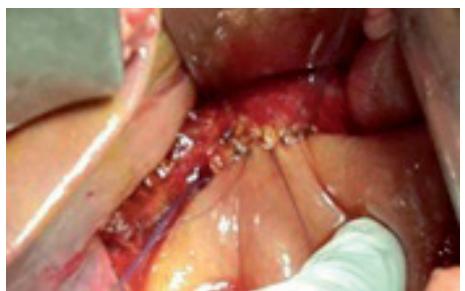

Figura 5. Anastomosis biliodigestiva terminada ( Hepatoyeyunoanastomosis en $Y$ de Roux).

Se realiza biopsia a tejido resecado el cual al corte histológicos muestra pared fibrosa y muscular lisa revestida por una mucosa de tipo columnar sin atipias con zonas extensamente esfaceladas y asociadas a congestión con microhemorragia en el corion subyacente; compatible con quiste coledociano. No se han encontrado cambios neoplásicos (figura 6).

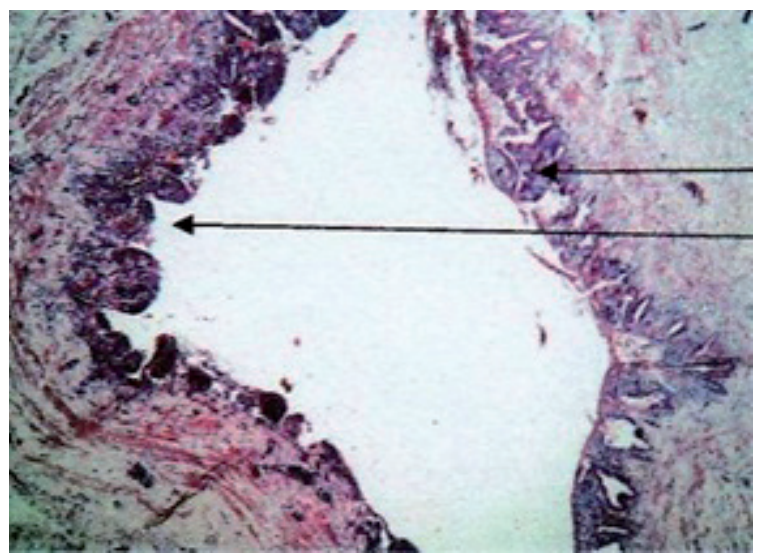

Figura 6. Corte histológico de muestra de resección quirúrgica.

La paciente evoluciona favorablemente en el posoperatorio bajo tratamiento clínico con hidratación parenteral, antibióticos y analgésicos, cuidados generales con buena tolerancia a la alimentación oral y niveles normales de enzimas hepáticas TCO 40 UI. TCP 55 UI, fosfatasa alacalina en 86 UI, amilasa de 60 UI y lipasa de 67 UI. Paciente se encuentra en buenas condiciones sistémicas por lo cual se le da el alta al sexto día del posoperatorio.

\section{DISCUSIÓN}

Se conoce poco sobre la etiología de los quistes del colédoco, incluso se discute si es una patología congénita o adquirida, siendo la teoría más aceptada la de las anomalías congénitas en la unión biliopancreática presentes en pacientes con quistes del colédoco. La unión del colédoco con el Wirsung antes de atravesar la pared duodenal, en un largo conducto común que favorece el reflujo hacia la vía biliar de las enzimas pancreáticas con la consecuente lesión y posterior daño y dilatación de la pared. ${ }^{1,8,13}$ Para otros esta formación quística estaría asociada al aumento de la presión en la vía biliar; sin embargo, hay casos en los que no se encuentra esta particularidad. ${ }^{8-10}$

Los quistes del colédoco habitualmente se diagnostican en la infancia, aunque un $20 \%$ de los cuadros se descubren en la edad adulta. El síntoma más frecuente es el dolor abdominal acompañado de la triada clásica: dolor, ictericia y masa abdominal, aunque no siempre suele debutar con estos síntomas y signos. ${ }^{1,9,13}$

Las pruebas de imagen, como la ecografía abdominal y tomografía axial computarizada(TAC) colaboran con el diagnóstico aunque no siempre con certeza, por lo que se considera que el diagnóstico preciso se realiza con la colangiopancreatografia retrograda endoscópica (CPRE) $)^{11,12}$ esta técnica permite clasificar el quiste, medir su tamaño, localización y sus relaciones anatómicas previamente a la cirugía. Actualmente la colangiorresonancia nuclear magnética (CRNM) puede sustituir a la CPRE, que en este caso fue la que permitió llegar al diagnóstico definitivo en esta paciente y poder plantear el tratamiento quirúrgico correspondiente, aunque la colangiorresonancia tiene sus beneficios más en niños, con diagnóstico de esta patología. Se trata de una exploración no invasiva y con menos complicaciones, aunque presenta la desventaja de no ser terapéutica. ${ }^{9,11}$

El tratamiento depende del tipo de quiste del colédoco. Los autores son unánimes en recomendar la cirugía con resección de los quiste de colédoco tipo I (figura 4). ${ }^{12,13}$ Se resuelve así el problema clínico y se previene la aparición de un colangiocarcinoma. La técnica quirúrgica más utilizada es la quistectomía con reconstrucción de la continuidad anatómica mediante una hepaticoyeyunoanastomosis en "Y de Roux", que fue la técnica que se aplicó en la caso de la 
paciente con exceresis completa del quiste y la restitución del tránsito biliar y digestivo. En los quistes tipo III la posibilidad de degeneración maligna es mucho más rara y en estos casos el tratamiento de elección es la esfinterotomía. Este gesto terapéutico permite el adecuado drenaje de la vía biliar y pancreática. .,11

La clasificación de Todani y colaboradores, muy aceptada para los quistes de colédoco, se basa en la morfología colangiográfica, ubicación y número de quistes intra y extrahepáticos del árbol biliar. El más frecuente es el tipo I y se limitan a las vías biliares extrahepáticas. Este a su vez se subdividen en IA que es una dilatación sacciforme, que compromete casi todo el conducto hepatocolédoco, el IB que es una dilatación sacciforme, que compromete solo a un segmento del conducto hepatocolédoco, que es el tipo que presentó la paciente del presente caso, como se puede ver en la imagen de la colangiografia y colangiorresonancia y el IC que es una dilatación difusa de todo el conducto hepatocolédoco. El tipo II que es un divertículo que afecta al conducto cístico y el tipo III que es un coledococele. El tipo IV presenta múltiples dilataciones quísticas de la vía biliar intra y extrahepática; aquí también se tiene dos subtipos: el IVa, que afecta solo a la via biliar intraheaptica; y el IVb, que afecta únicamente a la extrahepática. El tipo V, conocido también como enfermedad de Caroli, es una afectación difusa quística de la vía biliar intrahepática y asociada a fibrosis de la misma. ${ }^{2}$ (Figura 7)

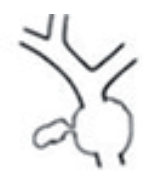

IA

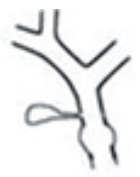

IB

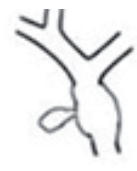

IC

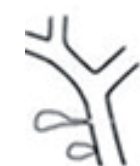

II
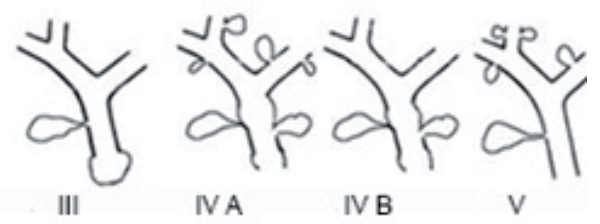

Figura 7. Clasificación del quiste de colédoco según Todani.²

CONCLUSIONES

El quiste de colédoco es una patología biliar congénita diagnosticada principalmente en la primera década de la vida. Cuando es identificada en pacientes adultos, el manejo es quirúrgico en la mayoría de los casos; el tratamiento debe incluir resección completa del quiste con anastomosis biliodigestiva. El drenaje interno hacia un asa del intestino delgado sin la resección del quiste, no es recomendable por la posibilidad de complicaciones infecciosas y probabilidad de daño crónico del hígado o el desarrollo de malignidad en el remanente del quiste.

En la experiencia de los autores, es posible el manejo quirúrgico con resección del quiste, con una morbilidad considerable pero con mortalidad baja. El reporte de este caso en particular es de suma importancia puesto que es una patología poco común, que puede pasar asintomática por muchos años y de tratamiento quirúrgico agresivo por su tendencia a la malignización del quiste del colédoco.

\section{REFERENCIAS BIBLIOGRÁFICAS}

1. E., García-Borobia F.J., Frago R., et al. Quistes de los conductos biliares del adulto: estrategia quirúrgica. Cir Esp. 2008 Nov; 84(5):256-61.

2. Todani T, Watanabe Y, Narusue M, Tabuchi K, Okajima K. Congeni-tal bile duct cysts. Classification, operative procedures, and review of thirty-seven cases including cancer arising from choledochal cyst. Am J Surg 1977;134:263-269.

3. Int Abstr Surg. Altet J., Rafecas A., Fabregat J., Ramos 2. . 1959 Jan; 108(1):1-30

4. Todani T, Watanabe Y, Toki A, Urushihara N. Carcinoma related to cho-ledochal cysts with internal drainage operations. Surg Gynecol Obstet 1987;164:61-64.

5. Lenriot JP, Gigot JF, Segol P, Fagniez PL, Fingerhurt A, Adloff M. Bile duct cysts in adults. A multi-institutional retrospective study. Ann Surg 1998;228:159-166.

6. Chijiiwa K, Koga A. Surgical management and long-term follow-up of patients with choledochal cysts. Am J Surg 1993;165:238-242.

7. Deziel DJ, Rossi RL, Munson JL, Braash JW, Silverman ML. Manage-ment of bile duct cysts in adults. Arch Surg 1986;121:410-415.

8. Stain SC, Guthrie CR, Yellin AE, Donovan AJ. Choledochal cyst in the adult. Ann Surg 1995;222:128133.

9. Lipsett PA, Pitt HA, Colombani PM, Boitnott JK, Cameron JL. Cho-ledochal cyst disease. A changing pattern of presentation. Ann Surg 1994;220:644652.

10. Chaudhary A, Dhar P, Sachdev A, Kumar N, Vij JC, Sarin SK, et al. Choledochal cysts differences in children and adults. Br J Surg 1996;83:186-188. 
11. Metcalfe MS, Wemyss-Holden SA, Maddern GJ. Management dilem-mas with choledochal cysts. Arch Surg 2003;138:333-339.

12. Alonso-Lej F, Rever WB Jr, Pessagno DJ. Congenital choledochal cyst, with a report of 2 and an analysis of 94 cases. Int Abstr Surg 1959;108:1-30.

13. Kasai M, Asakura Y, Taiba Y. Surgical treatment of choledochal cyst. Ann Surg 1970;172:844-851.

14. Hopkins NFG, Benjamin IS, Thompson MH, Williamson RCN. Com-plications of choledochal cysts in adulthood. Ann R Coll Surg Eng.
15. E., García-Borobia F.J., Frago R., et al. Quistes de los conductos biliares del adulto: estrategia quirúrgica. Cir Esp. 2008 Nov; 84(5):256-61.

16. Alvarado-García R, Reza-Villa A, Uribe-3. Ramos D, Gallego-Grijalva J. Quiste gigante de colédoco. Informe de un caso pediátrico. Cir Ciruj 2002; 70: 40-43. 